recommended surveillance for two years. They raise certain questions as to the length of time during which the syphilitic infection may be masked and the possibility of its transmission during the latent stage.

Of 183 cases of fresh untreated syphilis which I have seen, 10 patients gave a history which appeared to show an original double infection with syphilis and gonorrhoea from a single exposure, with no intervening exposure since their treatment with penicillin. The relevant details are given in the Table. Nine of the 10 cases had received 100,000 units of penicillin for the gonorrhoeal infection ; one case had received 60,000 units only. In all these cases a primary syphilitic lesion, in which $T$. pallidum was found, appeared within two months of the penicillin treatment.

The average time interval from penicillin treatment to the appearance of the ulcer in these cases was 38.9 days ; from the probable date of exposure it was $47 \cdot 2$ days.

One patient (No. 8) admitted with a primary ulcer of the tongue and left-sided submandibular adenopathy, stated that at the time at which he received treatment for gonorrhoea he had had "a sore on the tongue in the same place and a lump in the neck ", which had subsided after penicillin treatment only to recur a week before his admission. In view of the incubation period of the gonorrhoea, it is possible that his original lesion was the primary one with its satellite bubo, which had been aborted by penicillin.

It has been impossible to find in the literature any figures which would serve as a comparable control, but in 43 cases of primary syphilis, admitted over the same period, the average interval from the time of exposure to the appearance of the lesion was 39 days. I can only conclude that the amount of penicillin given may have delayed slightly the appearance of the lesions. The percentage of cases of primary syphilis in which there are positive serological tests for syphilis within one week of the appearance of the ulcer varies from 36 to 66 (Stokes). Among my cases, 5 out of 10 yielded a doubtfully or strongly positive Kahn test result. The administration of penicillin, therefore, does not appear to have caused any marked variation in the results of blood serological tests from that which would be expected.

\title{
Summary and conclusions
}

Ten cases of gonorrhoea treated with penicillin, in which syphilis subsequently developed from the same exposure, are described. In all the cases cutaneous lesions, which were positive on dark-field examination, developed within 2 months, and there was no apparent delay in the appearance of a positive serological reaction.

On the evidence submitted, it is suggested that surveillance of penicillin-treated cases of gonorrhoea to exclude syphilis does not require to be extended beyond the customary 3 months.

E. CRONIN, M.D. Captain, R.A.M.C.

\section{REFERENCES}

Lydon, F. L., and Cowe, W. R. S. (1945) Brit. med. J., 1, 110.

Shafer, Bertha, and Zakon, S. J. (1944) Arch. Derm. Syph., N.Y., 50, 200.

Stokes, J. H. (1934) Modern Clinical Syphilology, 2nd edition, London, p. 597.

\footnotetext{
Bismuth as sole medication in syphilis

Gaté and Cuilleret of Lyons (in a paper abstracted in Bruxelles-Médical for 26th August 1945) advocate the use of bismuth alone in the treatment of early syphilis. They point out that drug resistance is a very rare occurrence in bismuth medication and that the contra-indications are few. They claim, in the 14 years during which they have used bismuth exclusively, to have obtained results comparable with those of methods in which it is an adjuvant. What are called " paradoxical cases" occur, but in general the results of bismuth therapy are stated to be speedy, regular and prolonged, particularly as regards the prevention of impotence; complications are fewer than with the use of arsenicals and these drugs are said to be unnecessary, even in temporary association with bismuth.-La prophylaxie antivénérienne, April 1945.
} 